University of Wollongong

Research Online

Faculty of Engineering and Information

Faculty of Engineering and Information

Sciences - Papers: Part A

Sciences

$1-1-2015$

Calibrating Markov chain-based deterioration models for predicting future conditions of railway bridge elements

Niroshan Walgama Wellalage

University of Wollongong, wwnk807@uowmail.edu.au

Tieling Zhang

University of Wollongong, tieling@uow.edu.au

Richard Dwight

University of Wollongong, radwight@uow.edu.au

Follow this and additional works at: https://ro.uow.edu.au/eispapers

Part of the Engineering Commons, and the Science and Technology Studies Commons

Research Online is the open access institutional repository for the University of Wollongong. For further information contact the UOW Library: research-pubs@uow.edu.au 


\title{
Calibrating Markov chain-based deterioration models for predicting future conditions of railway bridge elements
}

\begin{abstract}
Existing nonlinear optimization-based algorithms for estimating Markov transition probability matrix (TPM) in bridge deterioration modeling sometimes fail to find optimum TPM values, and hence lead to invalid future condition prediction. In this study, a Metropolis-Hasting algorithm (MHA)-based Markov chain Monte Carlo (MCMC) simulation technique is proposed to overcome this limitation and calibrate the state-based Markov deterioration models (SBMDM) of railway bridge components. Factors contributing to rail bridge deterioration were identified; inspection data for 1,000 Australian railway bridges over 15 years were reviewed and filtered. The TPMs corresponding to a typical bridge element were estimated using the proposed MCMC simulation method and two other existing methods, namely, regression-based nonlinear optimization (RNO) and Bayesian maximum likelihood (BML). Network-level condition state prediction results obtained from these three approaches were validated using statistical hypothesis tests with a test data set, and performance was compared. Results show that the MCMC-based deterioration model performs better than the other two methods in terms of network-level condition prediction accuracy and capture of model uncertainties.
\end{abstract}

\section{Keywords}

chain, models, predicting, calibrating, future, markov, conditions, railway, bridge, elements, deterioration

\section{Disciplines}

Engineering | Science and Technology Studies

\section{Publication Details}

Walgama Wellalage, N. K., Zhang, T. \& Dwight, R. (2015). Calibrating Markov chain-based deterioration models for predicting future conditions of railway bridge elements. Journal of Bridge Engineering, 20 04014060-1-04014060-13. 


\title{
Calibrating the Markov Chain Based Deterioration Models for Predicting Future Conditions of Railway Bridge Elements
}

\author{
Niroshan K.Walgama Wellalage ${ }^{1}$, Tieling Zhang ${ }^{2}$, Richard Dwight ${ }^{3}$
}

\begin{abstract}
Existing nonlinear optimization based algorithms for estimating Markov Transition Probability Matrix (TPM) in bridge deterioration modelling sometimes fail to find optimum TPMs values and hence, leads to invalid future condition prediction. In this study, Metropolis-Hasting Algorithm (MHA) based Markov Chain Monte Carlo (MCMC) simulation technique is proposed to overcome the above limitations and calibrate the State Based Markov Deterioration Models (SBMDM) of railway bridge components. Contribution factors for rail bridge deterioration were identified and, the inspection data over 15 years of 1000 Australian railway bridges were reviewed and filtered. The TPMs corresponding to a typical bridge element were estimated by using proposed MCMC simulation method and two other existing methods, namely Regression based Nonlinear Optimization (RNO) and Bayesian Maximum Likelihood (BML) approaches. Network level condition state prediction results obtained from 3 different approaches were validated by using statistical hypothesis tests with a test data set, and performance was compared. Result shows that MCMC based deterioration model gives the best performance than the other two methods in terms of network level condition prediction accuracy and capturing the model uncertainties.
\end{abstract}

Keywords Markov Chain Monte Carlo Simulation, Metropolis-Hasting Algorithm, Railway Bridge Deterioration Modelling, Transition Probability Matrix

\section{Introduction}

There are approximately 15,000 bridges in Australia's rail network, which were made with different materials, constructed at different years. These bridges are inspected and maintained by separate organisations according to different inspection and maintenance standards. Furthermore, they are subject to different magnitudes, frequencies and distributions of rail loading and exposed to different environmental categories. According to Nielsen et al. (2012), the current inspection and maintenance practices within the Australian rail bridge industry doesn't have capability of predicting the future conditions of bridge components. Decision making procedure is subjective and it doesn't provide optimization for life cycle cost. Maintaining those thousands of bridges has become a real challenge for bridge engineers, and managing the rail infrastructure is a difficult task without having a systematic way for decision makings such as 'what' and 'when' to repair (Prasad et al., 2007). Currently research projects are undergoing to implement Bridge Management System (BMS) for rail bridges in Australia. However, the most suitable deterioration models which fit to the current inspection and maintenance regimes are vital parts

\footnotetext{
${ }^{1}$ School of Mechanical, Materials and Mechatronic Engineering, University of Wollongong, Australia

${ }^{2}$ School of Mechanical, Materials and Mechatronic Engineering, University of Wollongong, Australia

${ }^{3}$ School of Mechanical, Materials and Mechatronic Engineering, University of Wollongong, Australia
} 
in rail bridge asset management system and need to be developed based on historical inspection and maintenance data available. This is the motivation of this study.

Network level deterioration modelling is only considered here. The outcomes of network level deterioration models are the prediction of condition changes with time of the given bridge/component network and that can be used for planning maintenance including repair and rehabilitation activities of bridges and for calculating Life Cycle Cost (LCC) of bridges (Morcous, 2011). Bridge inspection condition rating data are often used for developing network level deterioration models. Although the deterioration processes of bridge components are continuous, discrete condition ratings are often used to measure the component condition in order to reduce the complexity of the continuous condition monitoring (Mandath, 1995). For example, condition scale of 1 to 6 is used by a large rail bridge management company in Australia. Condition rating 1 represents the best condition state without any visual defect and rating 6 indicates the completely failed condition of the bridge or component. Inspections are normally conducted once in every one or two years.

Bridge components deteriorate with time and deterioration rates and patterns may vary with different contributing factors such as age, rail-traffic volume (Tonnage passed on bridge for a given time), span, number of tracks, material type, functional classification (passenger train bridges or freight train bridges), nature of the defect, structure type and environmental categories, etc. These impact factors were identified based on most common contributing factors that were considered in previous studies (e.g., Radomski, 1994; Jiang, 1990; Scherer, 1994; Agrawal, 2009; Morcous et al., 2002) and by considering expert opinions of rail bridge engineers.

Markov chain approach is the most popular stochastic deterioration modelling technique that has been intensively used for predicting the future conditions of infrastructure facilities in network level (Agrawal, 2009). Many advanced Bridge Management Systems (BMS) in the world employ State Based Markov Deterioration Models (SBMDM). For example, PONTIS in USA, OBMS in Canada, KUBA in Switzerland, NYSDOT in New York and Quebec etc., (Manamperi and Lake, 2013; Devraj 2009; Roelfstra et al., 2004) are utilized for deterioration modelling. The main task of SBMDM is to estimate Transition Probability Matrixes (TPMs) from inspection data, which is also known as calibrating Markov models (Tran, 2007). TPM describes probabilities of state transitions from one condition state to another in a given inspection cycle. Stationary Markov model applies time independent transition probability matrix (TPM) by assuming a homogeneous deterioration pattern for a selected data set. By holding other potential contribution factors (as explained in the above paragraph) constant with age, it is possible to form different similar characteristic element groups to isolate and analyse deterioration process with time in network level (Veshosky, 1994). Deterioration pattern of similar characteristic component groups, without repair and treatment work in between, can be assumed to be homogeneous when limited condition rating data are available (Morcous, 2011) and deterioration process of those can be modelled by using stationary Markov models considering single TPM (Morcous, 2011).

There are different competitive and recommended methods in literature for calibrating (estimating TPM) Markov chain based infrastructure network-level deterioration models 
(Micevski et al., 2002; Hong et al., 2006; Agrawal, 2009; Morcous, 2011; Ranjith et al., 2013) with their own advantages and limitations, some of these methods have been applied to bridge deterioration modelling studies (Morcous, 2011; Ranjith et al., 2013; Agrawal, 2009) and only a few of them are used in existing Bridge Management Systems (BMS).

PONTIS in USA and KUBA in Switzerland use Regression analysis by using pairs of inspections for estimating TPM of homogeneous Markov models (Devraj, 2009; Roelfstra et al., 2004). PONTIS approach focuses on the error or difference between the predicted Markov conditions and the inspection based conditions. The sum of errors is calculated first, for all the inspection data used for estimation and, then formal minimization procedure is applied to find the appropriate transition probability values such that the sum of error is reduced to the minimum. Possible negative transition probabilities that can be found in the regression procedure, inaccurate matrix calculation with noninvertible matrixes and considering single TPM even deterioration process is inhomogeneous,can be considered as the drawbacks of PONTIS deterioration model (Devraj, 2009). In order to overcome these limitations, Devraj (2009) proposed a Regression based Non-linear Optimization (RNO) techniques to model the deterioration process with multiple TPMs. He compared results from his method with PONTIS approach; found that RNO with multiple TPMs has less relative error compared to PONTIS approach. Nebraska Department of Roads (NDOR) recently decided to adopt "PONTIS", (Morcous, 2011). Morcous (2011) recently analysed and estimated TPMs of Nebraska bridge components by using RNO method for homogeneous Markov models. Agrawal (2009) came up with a computer tool for calculating deterioration rates of New York State Department of Transportation (NYSDOT) bridges by using RNO based Markov deterioration models, and Weibull-based approach (Agrawal et al., 2010). He compared the results using Markov models and Weibull approach and concluded that Weibull based approach performs better than Markov model with a few case studies. However, It should be noted that for given initial condition, performance of Sate Based Markov models are solely dependent on the accuracy of the TPM andreliability of the techniques use to estimate it. RNO based Markov model calibration techniques explained in above studies sometimes fails to obtain optimum TPM vales due to subjective regression function type and inaccurate optimum points (Local optimum points) obtained through nonlinear optimization as explained in Section 3. Proposed MCMC approach is able to overcome above limitations and estimate more accurate TPM for bridge components in homogeneous Markov models. For effective management of bridge maintenance, inspection and rehabilitation, these TPMs can be fed into the BMS such as 'PONTIS' or used to counter check the TPM estimated through regressionor RNO methods. Other advantage of proposed methodology foe estimating TPM of SBMDM compared to other existing methods is capable of providing confidence intervals for TPM values.

\section{Review of the Infrastructure TPM Estimation Methods of SBMDM}

Markov Transition Probability Matrix (TPM) is often estimated in network level analysis by "segmenting infrastructure facilities into groups of homogeneous explanatory variables to capture the fact that transition probabilities are functions of these variables" (Agrawal, 2009). The variables involved in this paper are the contributing factors such as rail-traffic volume, 
number of tracks, material type, functional classification, environmental categories, etc. The most commonly used approach for estimating the transition probabilities is the regression based non-linear optimisation method (RNO) (Carnahan et al., 1987; Jiang et al., 1988; Veshosky et al., 1994; Morcous, 2006; Morcouset al., 2010 and $\mathrm{Bu}$ et al.,2012) by minimizing the absolute differences between the average condition states given by regression fitting curve and Expected Performance index. This method is used to estimate TPMs through closely mapping the expected condition index curve given by Markov chain model into the regression performance curve. However, the reliability of this approach solely depends on accuracy of the formula type selected for curve fitting analysis, which is often assumed to be a $3^{\text {rd }}$ order polynomial function (Agrawal et al., 2010; Jiang et al., 1988; Morcous, 2006; Morcous, 2011 and Bu et al., 2012). Furthermore, use of linear regression may not be appropriate (Madanath et al., 1995) since there are dependent variables in the system and infrastructure condition ratings are discrete and ordinal. Ranjith et al. (2013) proposed Bayesian Approach based Nonlinear Optimisation (BANO) method for estimating TPM of bridge elements in SBMDM; compared the performance with RNO and concluded that BANO performs well compared to RNO. However, all nonlinear optimisation techniques discussed in this section have some common drawbacks such as: It may stop at local minimum points resulting in incorrect transition probability values; it cannot provide confidence limits of the transition probabilities and it is difficult to update when new data are available (Tran, 2007).

Some researchers (Madanat et al., 1995; Bulusu et al., 2007) have used Ordered Probit Models (OPM) to establish TPM of bridge components. These models are also linear regression models and a continuous latent (unobserved) variable is used as the basis for the ranking of discrete data (Baik et al., 2006). One advantage of this method is its capability to link deterioration to relevant explanatory variables and able to capture non-stationary nature of the TPMs. These models consider unobservable amount of deterioration random variables and a linear function of explanatory variable vector with assumed standard normal errors. However, according to Tran et al. (2009), assumption of standard normal distribution for data error cannot be directly verified since the data error is incorporated in deterioration model and they fail the goodness-of-fit test in validation. Furthermore, Kallen (2010) convinced in many ways that Ordered Probit Models are not very suitable for estimating Markov chain transition probabilities after conducting extensive review of current OPMs for visual inspection data. Again, Madanat et al. (1995) proposed a negative binomial regression model for estimating TPMs. Shortcomings of this method are that the observed condition states are considered to be identically distributed and independent, inconsistency between noncumulative nature of the explanatory variables and the cumulative nature of continuous deterioration level used (Mishalani et al., 2002).

Bayesian approach (BA) based analysis methods have become more popular in recent years with modern development of computer based advanced sampling techniques (Martinez and Martinez, 2002). MCMC models are developed in Bayesian frame works has been widely used to estimating unknown parameters or posterior distributions in complex statistical models including deterioration models (Scott, 2000; Hong and Prozzi, 2006; Robert et al., 2000; Capper, 2005; Kobayashi et al., 2013; Micevski et al., 2002; Tran, 2007). There are considerable number of literature found in application of MCMC for parameter estimating of Hidden Markov models 
(Kobayashi et al., 2013; Scott, 2000; Hong and Prozzi, 2006; Robert et al., 2000) for deterioration modelling. Very few studies were undertaken to use MCMC for estimating TPM of SBMDM in pipe network modelling (Micevski et al., 2002; Tran, 2007). Micevski et al. (2002) successfully applied BA based Markov Chain Monte Carlo (MCMC) simulation method with Metropolis-Hasting Algorithm (MHA) to estimate TPMs of storm water pipe network deterioration models. Tran (2007) compared BA based MCMC method, Ordered Probit Models and BA based nonlinear optimization method for estimating TPMs of storm water pipe network deterioration models and concluded that BA based MCMC method with MHA is more suitable for calibrating pipe network Markov deterioration models. Furthermore, Tran (2007) argued that BA based MCMC method with MHA is able to solve the above issues and it can predict the future condition states of storm water pipes in network level more precisely compared to other nonlinear optimisation methods and Ordered Probit Models. However, MHA based MCMC program proposed by Tran (2007) has following limitations:

1. As convergence diagnostic procedure was not considered, the optimal number of iterations needed to run for obtaining stationary target distributions is unknown.

2. The run of simulation is forced to stop with some iterations at optimum acceptance rate point, 0.234 , may not be appropriate because the simulation may not have reached the convergence.

3. That Logistic function is used to generate the proposal random transition probability values may violate the assumption of symmetric proposal density required for using Metropolis algorithm or simplified MHA as shown in Eqs. (9) and (10) in Section 3.2.2.

This paper for the first time investigates MCMC method with powerful MHA algorithm in depth for bridge service condition prediction; with homogeneous State Based Markov Deterioration Models (SBMDM). Diagnostic procedure proposed by Gelman and Rubin (1992) was considered for convergence monitoring by considering multiple simulation results. Variance-Covariance matrix was tuned until acceptance rate becomes near to optimum value of acceptance 0.234 . Another function was developed to obtain multivariate normally distributed proposal transition probability values. Thus all three limitations above were eliminated and simulations were run to generate stationary distributions of transition probabilities.

Contribution factors for rail bridge deterioration were identified. Real network level inspection data of typical railway bridge component with similar characteristics (exposed to same contribution factors) were analysed by using proposed approach and two other existing methods, RNO and BAML; Statistical hypothesis tests were used to validate the suitability of models and, to compare the performances of the proposed model with that of RNO and BAML.

\section{Modelling Approaches}

Three modelling methods were selected for estimating Markov transition probabilities of railway bridge elements. These methods includeregression based nonlinear optimization, BA based maximum likelihood method and BA based MCMC method with MHA. MCMC method with 
MHA is a new application for bridge deterioration modelling but has been applied to pipe deterioration modelling (Tran, 2007; Micevski et al., 2002) previously. More details about these methods are explained in Section 3.2.2.

\subsection{Regression based nonlinear optimization method}

Performance Index, $\mathrm{PI}_{(t)}$, as a function of age, is obtained using regression curve fitting analysis on condition rating data after converted into PI values by $\mathrm{PI}=6-\mathrm{CR}$, where $\mathrm{CR}$ is a condition reading value. Then, TPM can be estimated by solving the constrained non-linear optimization problem (Jiang \& Sinha, 1989; Morcous, 2011) that minimizes the sum of absolute difference between the regression model $\mathrm{PI}_{(t)}$ and the expected performance index $\mathrm{EPI}_{(t)}$ which is also called as the objective function and can be expressed as a function of Transition Probability Matrix in Markov chain model analysis. If the condition state matrix after time $t$ is known, the expected value of facility condition at time $t$ can be calculated and defined as Expected Performance Index $\left(\mathrm{EPI}_{(t)}\right)($ Devraj, 2009).

$$
\operatorname{EPI}_{(t)}=\mathbf{C}_{(t)} \times \mathbf{S}
$$

where $\mathbf{S}$ is a column matrix composed of condition ratings and $\mathbf{S}$ is the transpose of matrix of [5 $\left.\begin{array}{llll}4 & 3 & 2 & 1\end{array}\right]$ if there are five condition status such as $1,2, \ldots, 5$ for instance; $\mathbf{C}_{(t)}$ is condition state matrix given in the following equation,

$$
\mathbf{C}_{(t)}=\mathbf{C}_{(0)} \times \mathbf{P}^{t}
$$

where $\mathbf{C}_{(t)}=\left[\begin{array}{llllll}\mathrm{C}_{1(t)} & \mathrm{C}_{2(t)} & \mathrm{C}_{3(t)} & \mathrm{C}_{4(t)} & \mathrm{C}_{5(t)}\end{array}\right]$ and $\mathrm{C}_{\mathrm{i}(t)}(i=1,2, \ldots, 5)$ is percentage of bridges/components in each condition rating after $t$ years; $\mathbf{C}_{(0)}=\left[\begin{array}{lllll}1 & 0 & 0 & 0 & 0\end{array}\right]$ is initial condition state vector at $t=0$ and $\mathbf{P}$ is TPM which can be deduced to the following format for "Do nothing" option in one time step, one year for example, as given in Morcus (2011)

$$
\mathbf{P}=\left[\begin{array}{ccccc}
p_{11} & 1-p_{11} & 0 & 0 & 0 \\
0 & p_{22} & 1-p_{22} & 0 & 0 \\
0 & 0 & p_{33} & 1-p_{33} & 0 \\
0 & 0 & 0 & p_{44} & 1-p_{44} \\
0 & 0 & 0 & 0 & 1
\end{array}\right] .
$$

However, in two-year TPM, multi state transition events will be automatically taken into account according to Markov chain property and can be calculated by using second power of one-year TPM (Morcus, 2011).

The objective function of this optimization problem is given below (Butt et al., 1987):

$$
\operatorname{minimize}\left(\sum_{t=1}^{t=T}\left|\mathrm{PI}_{(t)}-\mathrm{EPI}_{(t)}\right|\right)
$$

where $T=$ maximum age found in the data set. 


\subsection{Bayesian approach}

Suppose $\mathrm{Y}=\left\{\mathrm{y}_{1}, \mathrm{y}_{2}, \mathrm{y}_{3}, \ldots . . ., \mathrm{y}_{n}\right\}$ represents a set of condition rating data of one type of bridge element from a group of bridges and $\theta$ representsunknown model parameter vector (here, the unknown model parameters are elements $p_{i j}$ in TPM). The joint probability distribution $\mathrm{P}(\mathrm{Y} / \theta)$ is known as the sampling distribution or likelihood function which should be known to perform any inference (Martinez and Martinez, 2002). $\mathrm{P}(\theta / \mathrm{Y})$ is known as the posterior distribution or target distribution and $\mathrm{P}(\theta)$ is called prior distribution of unknown model parameter. According to Bayes' rule for known values of data $\mathrm{Y}$, the posterior distribution of model parameter is given by

$$
\mathrm{P}(\theta / \mathrm{Y})=\frac{\mathrm{P}(\theta, \mathrm{Y})}{\mathrm{P}(\mathrm{Y})}=\frac{\mathrm{P}(\theta) \mathrm{P}(\mathrm{Y} / \theta)}{\mathrm{P}(\mathrm{Y})}
$$

It is clear that $\mathrm{P}(\mathrm{Y})$ is not a function of model parameter $\theta$; and for fixed $\mathrm{Y}$, it can be considered as a constant which is known as normalizing constant. By omitting the normalizing constant in equation (5), the posterior distribution density is proportional to the product of priordistribution density and the likelihood functionas given in following

$$
\mathrm{P}(\theta / \mathrm{Y}) \propto \mathrm{P}(\theta) \mathrm{P}(\mathrm{Y} / \theta)
$$

The primary task for a specific application is to develop a model for $\mathrm{P}(\theta, \mathrm{Y})$ and perform required computation to estimate the target density, $\mathrm{P}(\theta / \mathrm{Y})$ (Martinez and Martinez, 2002). Noninformative prior density is a common case and can be assumed as uniformly distributed based on Bayes-Laplace "principle of insufficient reason" (Sorensen \& Gianola, 2002). As a result, posterior distribution density is proportional to likelihood function.

Likelihood function of unknown transition probability density of Markov model for a given bridge data set $\mathrm{Y}=\left\{\mathrm{y}_{1}, \mathrm{y}_{2}, \mathrm{y}_{3}, \ldots . . ., \mathrm{y}_{n}\right\}$ can be simplified by using joint probability theory and deduced into logarithmic form for easy computation (Micevski et al., 2002; Tran, 2007; Ranjith et al., 2013) as

$$
\log [\mathrm{L}(\mathrm{Y} / \theta)]=\sum_{t=1}^{T} \sum_{i=1}^{5} N_{i}^{t} \log \left(C_{i t}\right)
$$

where, $\mathrm{L}(\mathrm{Y} / \theta)$ is the likelihood to observe a condition rating data set $\mathrm{Y}=\left\{\mathrm{y}_{1}, \mathrm{y}_{2}, \mathrm{y}_{3}, \ldots \ldots, \mathrm{y}_{n}\right\}$ for a given bridge element in a bridge group with total of $n$ records; $t$ is the bridge element age in years; $T$ is the largest age found in the data set and $N_{i}^{t}$ is the number of elements in condition $i$ at year $t$; $C_{i t}$ is the probability in condition state $i$ at year $t$ and can be expressed as a function of TPM by equation (2).

\subsubsection{Bayesian Maximum Likelihood method}

Tran (2007) used non-linear optimization techniques to estimate TPM of storm water pipe networks by maximizing the likelihood function given in equation (7) which is proportional to posterior distribution 
with non-informative uniform prior assumption as mentioned before. The relevant objective function and constraints are given as follows

$$
\operatorname{maximize} \log [\mathrm{L}(\mathrm{Y} / \theta)]=\operatorname{maximize} \sum_{t=1}^{T} \sum_{i=1}^{5} N_{i}^{t} \log \left(C_{i t}\right)
$$

for $i, j=1,2,3,4,5$ and $\sum_{t=1}^{5} p_{i j}=1$.

\subsubsection{MCMC simulation method with MHA}

Metropolis-Hasting Algorithm (MHA) is popularly applied to MCMC simulation and has been increasingly used in recent 20 to 30 years for simulating complex, nonstandard multivariate distributions (Brooks et al., 2011). It was the one of top ten algorithms used in the $20^{\text {th }}$ century (SIAM News, 2000) and recently used for many engineering applications (Micevski et al., 2002; Tran, 2007; Zhang et al., 2013; Onar et al., 2007; Yin et al., 2011). As given in equation (7), posterior distribution density of transition probabilities is proportional to multiple of prior distribution density into likelihood function. This property can be used to generate sample from posterior distribution by MCMC method with Metropolis Hasting Algorithm (MHA). When applying MHA, it's required to choose a proposal density $\mathrm{q}(x, y)$ where $\int q(x, y) d y=1$, for sampling from the target distribution (Brooks et al., 2011). Although, proposal distribution $\mathrm{q}(x, y)$ is arbitrarily chosen from some family distributions, performance is dependent on how much the selected distribution approximates the posterior (Roberts et al., 2001).

Let us consider a target distribution with density $\pi$. For given $\mathrm{X}_{n}, \mathrm{Y}_{n+1}$ (proposal value) is generated from some preferred density $\mathrm{q}\left(\mathrm{X}_{\mathrm{n}}, y\right)$ and is then accepted with probability $\alpha\left(\mathrm{X}_{n}, \mathrm{Y}_{n+1}\right)$ which is given by

$$
\alpha(x, y)= \begin{cases}\min \left\{\frac{\pi(y)}{\pi(x)} \frac{\mathrm{q}(y, x)}{\mathrm{q}(x, y)}, 1\right\}, & \pi(x) q(x, y)>0 \\ 1, & \pi(x) q(x, y)=0 .\end{cases}
$$

If the proposed value is accepted, set $\mathrm{X}_{n+1}=\mathrm{Y}_{n+1}$; otherwise, set $\mathrm{X}_{n+1}=\mathrm{X}_{n}$. The proposal density $\mathrm{q}(x, y)$ is often chosen from a symmetric and multivariate distribution which is also known as symmetric Random-Walk Metropolis algorithm (RWM). Since $\mathrm{q}(y, x)=\mathrm{q}(x, y)$ in this case, acceptance probability in equation (9) can be further simplified (Chib et al., 1995) into

$$
\alpha(x, y)=\min \left\{1, \frac{\pi(y)}{\pi(x)}\right\}
$$

According to Large sample theory, the posterior distribution of the parameters often approaches a multivariate normal distribution (Gelman et al., 2004; Schervish, 1995). For this study also, Multivariate Normal Density (MVD) was chosen as the proposal density for ease of implementation. Initial values of $p_{i j}$ for MHA were randomly chosen between 0 and 1 since they do not affect the convergence to the target distribution of the chain according to the theory (Brooks, 1998). Values for Variance-Covariance matrix in proposal density can be assumed initially. However, if assuming extremely small values for Variance-Covariance matrix, it will result in a long convergent time with high acceptance rate. On the other hand, if Variance- 
Covariance matrix is assumed to contain larger values, the Markov chain can get stuck in several places and ended up with a low acceptance rate (Brooks et al., 2011). Therefore, the VarianceCovariance Matrix (VCM) for MVD was arbitrarily chosen and tuned until the acceptance rate becomes near to the optimum value of acceptance, 0.234 (Roberts et al., 2001).

There are several methods in literature for deciding when to stop iterations in MCMC simulations (Martinez and Martinez, 2002). The convergence diagnostics of Gelman and Rubin (1992) is the one of the most popular methods in the statistical community and it can be used in any of the MCMC algorithms (Cowles, M. K., \& Carlin, B. P., 1996). Gelman and Rubin's coefficient (called as Estimated Potential Scale Reduction) was estimated by using results of 4 independent MCMC Chains, for each transition probability value. Gelman et al. (1995) recommended that the sequences to be run until all scalar factors become less than 1.1 or 1.2; otherwise, run the chain again with more iterations. In this study, simulations had to be run for 50000 iterations to reach the Estimated Potential Scale Reduction factors below 1.1 for all four parameters.

After generating sample distributions for transition probability values using MHA, the point estimators and confidence intervals for the transition probability values can be calculated. Although the posterior distribution of the TPM values may not be normally distributed, its sample distribution is considered as normal. Then the Centre Limit Theory can be used with unbiased point estimator for the mean values of the TPM to calculate $95 \%$ confidence intervals of TPM.

\section{Case Study}

\subsection{Bridge grouping}

Bridges can be classified into groups that have similar performance characteristics. Rail bridge performance is dependent on factors such as passenger rail-traffic volume (Tonnage passed per week), freight rail-traffic volume (Freight train load passed per week), Span, number of tracks, material type, functional classification (passenger train bridges or freight train bridges), structure type and environmental categories. These factors were identified according to bridge engineers' feedback and common factors considered in the past bridge deterioration studies (Radomski,1994; Jang, 1990; Scherer, 1994; Agrawal, 2009; Morcous et al., 2002; Agrawal, 2009; Le and Andrews, 2013). Therefore, bridges are grouped according to the following factors:

\section{a. Superstructure material}

Concrete, Steel, Timber, Concrete-in-rail, etc.

b. Number of railway tracks on bridge

For example, 1, 2, 3, etc.

c. Average tonnage passed per week
A: $\operatorname{Load} \leq 25000$
B: Load > 25000 


\section{d. Functionality}

- Passenger train bridges

- Freight train bridges

- Passenger+ Freight trains bridges

e. Environmental Categories

- Costal railway lines (railway lines near to coastal area)

- Inland railway lines (railway line away from the coastal areas)

\section{f. Element type}

Transoms, slab deck, primary beams, secondary beams, bearings, etc.

\section{g. Span length}

Short, medium and long span

\subsection{Data review, limitations and filtration}

Bridge condition data about 1000 bridges were collected and reviewed. All these bridges are located in state of Victoria and currently maintained by one of the major railway bridge organizations in Australia. Although most of bridges are relatively old, only 15 years back inspection records are currently available within the organization. For example, in some typical rail bridge lines, $99 \%$ of the superstructures and substructures are more than 60 years old and 15 years back inspection records represent condition rating data from 45 years old or more. Even for the existing inspection recodes of 15 years back, some of the major bridge components were not monitored regularly. However, some bridge components such as timber bridge decks, current ages spread between 0 and 80 years and some inspections, maintenance and repair records are available for developing MCMC based network level Markov chain deterioration models. Among 1000 bridge structures, single track bridges are dominant than multi-track bridges and most of them are subject to passenger train loading. There were 449 single track and passenger train loading bridges found in the data base. These bridges were classified according to the deck material type and given in Figure 1.

(Figure 1 is about here)

According to data availability and consideration of restricting to other contribution factors (Average tonnage passed per week, Environmental Categories, etc.), condition rating data over 15 years of 40 transverse timber bridge deck transoms in inland railway lines in Victoria state were selected to do the analysis in this paper. These transoms belong to the bridge group with similar characteristics in terms of contribution factors such as Superstructure material is Steel, Number of railway tracks on bridges is 01, Average tonnage passed per week is Category A, Functionality is Passenger train bridges, Environmental Category is Inland railway lines and Span is Short span. 
A major problem identified from data analysis is that the ratings are dependent on subjective nature of bridge inspectors. Veshosky (1994) argued that if same bridge component is rated by different inspectors, it potentially could result in different values. This problem has been addressed to some extent by conducting workshops and trainings for inspectors, reviewing and adjusting the condition rating data by supervisor and/or by conducting detailed inspections by experienced engineers (Veshosky, 1994). In general, all bridges in a one railway line and in the same state are inspected by one inspector. Thus, selecting condition rating data of bridges in one railway line can help to minimize the subjective nature in condition ratings. On the other hand, the number of available bridges for selection in one railway line is not large enough for doing analysis at network level. Therefore, in this study, analysis has to be done by combining bridges in different railway lines with similar characteristics based on assumption that the observed bridge condition ratings are randomly distributed about their true values.

Deterioration models in this study were developed for railway timber bridge decks with no improvement works being undertaken in between inspections. Therefore, condition rating data for any bridge deck element after repair and reconstruction actions, has been removed from the input datasheet. However, it has been found that the repair and maintenance work has not been all recorded appropriately. Some Bridge deck ratings figured condition improvement with time, for instance. It can be argued that, unless repair or maintenance work was done, bridge components would have been gradually deteriorating so that the bridge component's condition rating is either unchanged or increased into a larger number (Bolukbasi et al., 2004). Relying upon this assumption, bridge deck element whose condition rating was improved over the years were identified and also removed from the input datasheet. For some components, the smallest condition rating values have been observed even for relatively old bridge decks elements. The reason may be that repair or reconstruction works had not been recorded before 15 years, since the bridge agency has only 15 years back inspection records. Moreover, it has been also identified that some of the newly constructed bridge components had not been assigned into one condition rating category. Veshosky (1994) further stated that this could be due to inadequate initial design or construction or misapplication of rating procedure by bridge inspectors. Other avoidable data points were found, that was due to double counting of the condition rating of the same record. Therefore these unusual condition rating data were filtered out and rejected from the input datasheet. There are total of 242 records obtained after filtration for statistical validation and comparison purpose. The entire data set was split randomly into two groups of which one sub dataset contains $75 \%$ of the entire data as the calibrated data set and the rest as the test data set. The calibrated data set was used to build the model and the predicted analysis results were compared with the test data set to verify the model based on the $\chi^{2}$ test.

\subsection{Analysis results}

MTLAB Curve fitting toolbox was used for regression analysis. MATLAB codes were developed for objective function and constraint functions for nonlinear optimization. Optimization tool box was utilized to obtain the global minimum of objective function in constrained nonlinear optimization. The program was run with different initial values and using two algorithms of Interior-point \& Active-set until it converged to the same transition probability values to minimize stoppages at local minimums of objective function. 
MHA algorithm for MCMC was coded in MATLAB program. The Metrapolis samplers were generated with 50,000 iterations for the calibration data set and later for the entire data set until the transition probability values converged to stationary distributions. 15000 iterations in burn-in period were considered for warm-up runs. Variance-Covariance Matrix (CVM) was tuned until acceptance rate becomes near to the optimum acceptance rate of 0.234 (Robert \& Rosenthal, 2001; Tran, 2007).

\subsubsection{Regression based Nonlinear Optimization (RNO) method for calibrated data set}

Curve fitting regression analysis results of $3^{\text {rd }}$ order polynomial fit for selected data set is given in Figure 2. Nonlinear optimization objective function values for the generated transition probabilities with different number of iterations are displayed in Figure 3. Estimated 1 year and 2 years Transition Probability Matrixes (TPMs) are given in equations (11) and (12). Based on the TPMs, the condition percentage of each condition state $v s$. age can be predicted by using Markov chain model given in equation (2), as plotted in Figure 4.

(Figure 2 is about here)

(Figure 3 is about here)

$$
\mathrm{P}=\left[\begin{array}{ccccc}
0.85 & 0.15 & 0 & 0 & 0 \\
0 & 0.93 & 0.07 & 0 & 0 \\
0 & 0 & 0.93 & 0.07 & 0 \\
0 & 0 & 0 & 0.90 & 0.10 \\
0 & 0 & 0 & 0 & 1
\end{array}\right]
$$

$$
\mathrm{P}=\left[\begin{array}{ccccc}
0.7225 & 0.2670 & 0.0105 & 0 & 0 \\
0 & 0.8649 & 0.1302 & 0.0049 & 0 \\
0 & 0 & 0.8649 & 0.1281 & 0.0070 \\
0 & 0 & 0 & 0.8100 & 0.1900 \\
0 & 0 & 0 & 0 & 1
\end{array}\right]
$$

(Figure 4 is about here)

Figure 4 describes network level bridge deterioration model output which is the percentage of bridge components (Transoms) in each condition state along with age (C1 to $\mathrm{C} 5$ are the percentage curves of condition states 1 to 5 ) for the selected component group. According to $\mathrm{C} 1$ 
curve, it is clear that condition state one value approaches to zero (well below 5\%) after 30 years of age. Between age 5 and 22, C2 is the highest compared to the other condition states. Highest number of Transoms is in condition rating 3 between age 22 and 31 years. Percentage of C5 components continuously increases with time and records largest value after 31 years without any repair or rehabilitation work in between. At age $60,80 \%$ of the components will be in condition state 5 while all the components in other condition states become $10 \%$ or less.

\subsubsection{Bayesian Maximum Likelihood (BML) method for calibrated data set}

By using BML method, the estimated 1 year and 2 years Transition Probability Matrixes (TPMs) are given in equations (13) and (14). Based on the TPMs, the condition percentage of each condition state $v s$. age can be predicted by using Markov chain model given in equation (2), and can be plotted, similar to Figure 4.

$$
\begin{aligned}
& \mathrm{P}=\left[\begin{array}{ccccc}
0.86 & 0.14 & 0 & 0 & 0 \\
0 & 0.92 & 0.08 & 0 & 0 \\
0 & 0 & 0.92 & 0.08 & 0 \\
0 & 0 & 0 & 0.92 & 0.08 \\
0 & 0 & 0 & 0 & 1
\end{array}\right] \\
& \mathrm{P}=\left[\begin{array}{ccccc}
0.7396 & 0.2492 & 0.0112 & 0 & 0 \\
0 & 0.8464 & 0.1472 & 0.0064 & 0 \\
0 & 0 & 0.86464 & 0.1472 & 0.0064 \\
0 & 0 & 0 & 0.8464 & 0.1536 \\
0 & 0 & 0 & 0 & 1
\end{array}\right]
\end{aligned}
$$

\subsubsection{MCMC simulation with MHA results for calibrated data set}

MCMC simulation with MHA was programmed under MATLAB environment. By running this program with the input data as discussed in Section 4.2, the trace plots after 20000 iterations with no warm-up runs for $p_{11}, p_{22}, p_{33}$ and $p_{44}$ are shown in Figure 5. It is clear that all transition probability values are approximately convergent to their stationary distributions, respectively, after around 1000 iterations. Trace plots with 50000 iterations after 15000 burnings (Warm-up runs) are given in Figure 6. As given in Table 2 in Section 4.4, standard deviation for each transition probability, $p_{11}, p_{22}, p_{33}$ and $p_{44}$ values were found to be very small. The mean of each TPM element values is convergent to a constant value as shown in Figure 7. Figure 8 describes generated sample distributions for $p_{11}, p_{22}, p_{33}$ and $p_{44}$. Mean transition probability values obtained for 1 year and 2 years transition probability matrixes are shown in equations (15) \& (16), respectively. By using estimated TPM and known initial condition state vector, Markov chain model equation (2) was utilized to obtain the future condition state proportions. The percentage distribution of each condition sate vs. age can be plotted similar to Figure 4. 
Convergence diagnostic characteristics for all four variables are shown in Figure 9 from 2000 to 50000 iterations' chains. It's clear that after 50 thousand iterations all four Potential Scalar Reduction (PSR) factors are well below the 1.1 hence Gelman and Rubin (1992)'s convergence diagnostic conditions are satisfied by this MCMC model.

(Figures $5 \sim 9$ are about here)

$$
\begin{aligned}
& \mathrm{P}=\left[\begin{array}{ccccc}
0.86 & 0.14 & 0 & 0 & 0 \\
0 & 0.92 & 0.08 & 0 & 0 \\
0 & 0 & 0.92 & 0.08 & 0 \\
0 & 0 & 0 & 0.91 & 0.09 \\
0 & 0 & 0 & 0 & 1
\end{array}\right] \\
& \mathrm{P}=\left[\begin{array}{ccccc}
0.7396 & 0.2492 & 0.0112 & 0 & 0 \\
0 & 0.8464 & 0.1472 & 0.0064 & 0 \\
0 & 0 & 0.8464 & 0.1464 & 0.0072 \\
0 & 0 & 0 & 0.84281 & 0.1719 \\
0 & 0 & 0 & 0 & 1
\end{array}\right]
\end{aligned}
$$

\subsection{Validation and comparison}

Model performance was assessed by using Goodness-of-fit test using Chi-squared test statistics $\left(\chi^{2}\right)$ which is based on null hypothesis that the observed number of bridge elements is matched with the predicted number of elements in different condition states (Micevski et al., 2002; Tran, 2007; Ranjith et al., 2013). This test was applied to 3 deterioration modelling methods used in this study. $95 \%$ confidence level was used to evaluate the fitness of the models. Chi-squared test statistics $\left(\chi^{2}\right)$ for bridge element deterioration models in this study was calculated according to equation (17). Accuracy of $\chi^{2}$ statistics can be ensured by using one rule of thumb that is the predicted number of bridge elements in any condition $i$ must be not less than 5 (Micevski et al., 2002). $\chi^{2}$ values obtained for 3 deterioration models are given in Table 1.

$$
\chi^{2}=\sum_{i=1}^{5} \frac{\left(O_{i}-P_{i}\right)^{2}}{P_{i}}
$$

where, $O_{i}$ is observed number of transoms in condition $i$ (transom is one element of railway bridge deck), $P_{\mathrm{i}}$ is predicted number of transoms in condition $i$.

(Table 1 is about here) 
As expected, $\chi^{2}$ value of calibrated data set in each model is less than that of the test data set since the model parameters in TMPs such as those in equations (13) to (16) are determined by minimizing the mean square error for calibration data set. The calibration data set takes $75 \%$ of the entire data set and the test data set takes only $25 \%$. According to estimated values given in Table 1, all three models pass the goodness-of-fit test for calibrated and test data set since the obtained $\chi^{2}$ values are much less than the critical Chi-square value $\left(\chi_{0.05,4}^{2}\right)$ of 9.49 in this study. Therefore, it can be argued that those models are suitable for network level deterioration modelling of selected bridge elements in this study. Among the suitable three deterioration models, Bayesian MCMC simulation with MHA has the lowest Chi-square value which suggests that the MCMC method is the best calibration technique for the Markov chain model in this case study, followed by the Bayesian maximum likelihood method.

\subsection{MCMC simulation with MHA for entire data set}

By using the entire data set, the TPMs for 1 year and 2 years' time period are obtained as given in equations (18) and (19), respectively. Each value shown in equations (18) and (19) is the mean value of transition probabilities in TPM. These mean values and their $95 \%$ confidence limits for the entire data set are given in the Table 2. It can be argued that, since the exact values of transition probabilities are unknown, mean values with $95 \%$ confidence interval limits can be used to approximate the true values and the risk that the true value is not in the $95 \%$ confidence interval limits is $5 \%$. This also represents the uncertainty level in the estimation. Based on the 1 year TPM, the condition percentage of each condition state $v s$. age can be predicted by using Markov chain model given by equation (2) and plotted in Figure 10. It clearly shows that condition rating 1 and 2 are the dominant condition states in the first 10 years. After 30 years, however, condition state 5 becomes dominant. This is because the components with repair and rehabilitations were not included in the modelling in this study. On the other hand, $50 \%$ of the elements which are bridge deck transoms in this case study need to be repaired or replaced up to a service life of 40 years.

$$
\begin{aligned}
& \mathbf{P}=\left[\begin{array}{ccccc}
0.864 & 0.136 & 0 & 0 & 0 \\
0 & 0.921 & 0.079 & 0 & 0 \\
0 & 0 & 0.923 & 0.077 & 0 \\
0 & 0 & 0 & 0.913 & 0.087 \\
0 & 0 & 0 & 0 & 1
\end{array}\right] \\
& P=\left[\begin{array}{ccccc}
0.7465 & 0.2428 & 0.0107 & 0 & 0 \\
0 & 0.8482 & 0.1457 & 0.0061 & 0 \\
0 & 0 & 0.8519 & 0.1414 & 0.0067 \\
0 & 0 & 0 & 0.8336 & 0.1664 \\
0 & 0 & 0 & 0 & 1
\end{array}\right]
\end{aligned}
$$


(Figure 10 is about here)

(Table 2 is about here)

\section{Conclusions}

This paper presents a Metropolis-Hasting ( $\mathrm{MH}$ ) algorithm based Markov Chain Monte Carlo (MCMC) simulation approach to calibrating Markovian bridge deterioration models, considering limited inspection data available on Australian Railway bridges.

The developed approach and other two existing calibration methods, Regression Based Nonlinear Optimization (RNO) and Bayesian Maximum Likelihood (BML) estimation, were used to estimate TPM of timber deck transoms of railway bridges using inspection data for 15 years. Although the estimated TPMs are slightly different using these three approaches, the Bayesian MCMC simulation with MHA developed in this paper shows the best performance with lowest Chi-square value. It is observed that Non-linear optimization method sometimes stops at local minimums leading to inaccurate prediction of TPMs for selected bridge element. On the other hand, the outcome of proposed model based on Bayesian MCMC simulation with MHA is sample distributions of transition probabilities which increase the chance of capturing the true global optimum compared to RNO \& BML methods and it is capable of capturing the model uncertainties by providing confidence intervals for TPMs. Therefore, it is suggested that for estimating TPMs in homogeneous Markov bridge deterioration modelling, MCMC method is more powerful and accurate compared to the other two methods.

Another contribution of this paper is that the limitations on using MHA algorithm for generating stationary transition probability distributions in previous MCMC based pipe network deterioration models have been identified. These limitations have been overcome in the newly developed algorithm to ensure that the global optimum solutions can be found for the Markov transition probability values. Furthermore, in order to obtain stationary transition probability distributions, the convergence diagnostic procedure is used, which however were not considered in previous MCMC based deterioration modelling approaches for pipe network.

The approach developed in this paper is intended to apply for estimating TPM of other railway bridge components and to be employed in the new railway Bridge Management Systems (BMS) in the future in Australia. On the other hand, the approach developed can be integrated into a BMS such as "PONTIS" for counter checking of the regression based TPM estimations or as an alternative method for estimating TPM to improve the life cycle cost estimation of bridges.

\section{Acknowledgements}

This research was supported by CRC for Rail Innovation Australia under Project R3.118: "Life Cycle Management of Railway Bridges". 


\section{References}

Agrawal, A.K. (2009). Bridge Element Deterioration Rates Final Report, Transportation Infrastructure Research Consortium.New York State, Department of Transportation, USA.

Baik, H.S., Jeong, H.S., and Abraham, D.M. (2006)."Estimating transition probabilities in Markov chainbased deterioration models for management of wastewater systems."Journal of Water Resources Planning and Management, 132(1), 15-24.

Bolukbasi, M., Mohammadi, J., and Arditi, D. (2004)."Estimating the future condition of highway bridge components using National Bridge Inventory data.”Pract.Period.Struct.Des. Constr., 9(1), 16-25.

Brooks, S.(1998). "Markov Chain Monte Carlo Method and Its Application.”Journal of The Statistician, 47, 69-100.

Brooks, S.,Gelman, A. ,Jones, G., Meng, X. (2011). Handbook of Markov Chain Monte Carlo, Chapman $\&$ Hall/CRC, US.

Bu, G.,Lee, J.,Guan, H.,Blumenstein, M.,Loo, Y. (2012).“Development of an Integrated Method for Probabilistic Bridge Deterioration Modelling.”J. Perform. Constr. Facil., 10.1061/ (ASCE)CF.19435509.0000421.

Bulusu, S.,and Sinha, K.C. (1997).“Comparison of methodologies to predict bridge deterioration.” Transportation Research Record, 1597, 34-42.

Butt, A.A., Shahin, M.Y., Feighan, K.J., Carpenter, S.H. (1987)."Pavement performance prediction model using the Markov process."Transportation Research Record, 1123, Transportation Research Board, Washington, D.C., 12-19.

Carnahan, J., Davis, W., Shahin, M., Keane, P., Wu, M. (1987).“Optimal maintenance decisions for pavement management." Journal of Transportation Engineering, 113(5), 554-572.

Capper, O., Moulines, E., Ryden, T., 2005. Inference in hidden Markov models. Springer.

Cowles, M. K., \& Carlin, B. P. (1996). Markov chain Monte Carlo convergence diagnostics: A comparative review. Journal of the American Statistical Association, 91, 883-904.

Devraj, D. (2009). Application of non-homogeneous Markov chains in bridge management systems.Ph.D. thesis, Department of Civil and Environmental Engineering Wayne State University, USA.

Frangopol, D., Kallen, M., and Noortwijk, J. (2004)."Probabilistic models for life-cycle performance of deteriorating structures: review and future directions." Progress in Structural Engineering and Materials, 6(4), 197-212.

Gelman, A.,Carlin, J., Stern, H., Rubin, D. (2004). Bayesian Data Analysis, $2^{\text {nd }}$ Edition, London: Chapman $\&$ Hall.

Gelman, A. and Rubin, D.B. (1992), "Inference from Iterative Simulation Using Multiple Sequences(with discussion)." Statistical Science, 7, 457-511.

Hong, F., and Prozzi, J. A. (2006).“Estimation of Pavement Performance Deterioration UsingBayesian Approach.”Journal of Infrastructure Systems, ASCE, 12(2), 77-86.

Isaacson, D., and Madsen, R. (1976).Markov Chains, Theory and Applications,Wiley, New York.

Jiang, Y. \& Sinha, K. C. (1989).Bridge service life prediction model using the Markov chain. Transportation Research Record, 1223, 24-30. 
Jiang, Y., \& Sinha, K. C. (1989). The development of optimal strategies for maintenance, rehabilitation and replacement of highway bridges, Final report Vol. 6: performance analysis and optimization (Report No. FHWA/IN/JHRP-89/13). Purdue University.

Jiang, Y. (1990). The development of performance prediction and optimization models for bridge management systems. Ph.D. thesis, Purdue University, West Lafayette, IN.

Kallen, M.J. (2010). “A comparison of statistical models for visual inspection data.”Safety, Reliability and Risk of Structures, Infrastructures and Engineering Systems - Furuta, Frangopol \& Shinozuka (eds), Taylor \& Francis Group, London, pp. 3235-3242.

Le, B., and Andrews, J. (2013).“Modelling railway bridge asset management.”Journal of Rail and Rapid Transit, 227(4).

Lounis, Z., and Madanat, S.M. (2002)."Integrating mechanistic and statistical deteriorationmodels for effective bridge management." $7^{\text {th }}$ ASCE International Conference on Applications of Advanced Technology in Transportation, Boston, MA., U.S.A, pp. 513-520.

Madanat, S., Mishalani, R.,Wan Ibrahim, W. H. (1995). "Estimation of infrastructure transition probabilities from condition rating data."J. Infrastruct. Sys., ASCE, 1(2), 120-125.

Manamperi P., Lake N., (2013). “Bridge Management Using Performance Models", Project No. AT1537, Austroads Ltd 2013, AUS.

Martinez, W. L., and Martinez, A.R. (2002).Computational Statistics Handbook with MATLAB 2002. Chapman \& Hall/CRC, USA.

Micevski, T.,Kuczera, G., Coombes, P. (2002).“Markov Model for Storm Water Pipe Deterioration.” Journal of Infrastructure Systems, ASCE, 8(2), 49-56.

Mishalani, R. G.,and Madanat, S. M. (2002).“Computation of Infrastructure Transition Probabilities Using Stochastic Duration Models.”Journal of Infrastructure Systems, ASCE, 8(4), 139-148.

Morcous, G., Rivard, H.,Hanna, A. (2002). "Case-based reasoning system for modelling infrastructure deterioration."J. Comput. Civ.Eng., 16(2), 104-114.

Morcous, G. (2006).“Performance prediction of bridge deck systems using Markov chains.” Journal of Performance of Constructed Facilities, 20, 146-155.

Morcous, G.,and Hatami, A. (2011).Developing deterioration models for Nebraska bridges. Final report, Nebraska Department of Roads, pp. 182.

Nielsen, D.,Chattopadhyay, G.,Dhamodharan, R. (2012). "Life cycle management of railway bridges: defect management."Conference on Railway Engineering, 10-12 September 2012, Brisbane, Australia, p.425-434.

Onar, A., Thomas, F., Choubane, B., Byron, T. (2007). "Bayesian Degradation Modeling in Accelerated Pavement Testing with Estimated Transformation Parameter for the Response." J. Transp. Eng., 133(12), 677-687.

Prasad, P., Bridgwood, M., Coe, D. (2007). "Implementation of a Bridge Management System with the Australian Rail Track Corporation." 2nd Australian Small Bridges Conference, Gold Coast, Queensland, Australia.

Ranjith, S.,Setunge, S.,Gravina, R.,Venkatesan, S. (2013). "Deterioration Prediction of Timber Bridge Elements Using the Markov Chain.” J. Perform. Construction Facility, 27(3), 319-325.

Radomski, W. (2002). Bridge Rehabilitation, First Edition.

Roberts, G. O.,and Rosenthal, J. S. (2001).“Optimal Scaling for Various Metropolis-Hastings Algorithms.” Journal of Statistical Science, 16(4), 351-367.

Robert, C., Ryden, T., Titterington, D.M.,2000. Bayesian inference in hidden Markov models through the reversible jump Markov chain monte carlo method. Journal of the Royal Statistical Society Series B, Vol.62, 57-75. 
Roelfstra, G., Hajdin, R., Adey, B., and Brühwiler, E. (2004). "Condition Evolution in Bridge Management Systems and Corrosion-Induced Deterioration." J. Bridge Eng., 9(3), 268-277.

Ross, M. S. (1972). Introduction to Probability Models.McGraw-Hill, New York.

Schervish, M. J. (1995). Theory of Statistics (Section 7.4).New York: Springer-Verlag.

Scherer, W., and Glagola, D. (1994)."Markovian models for bridge maintenance management.”Journal of transportation engineering, 120(1), 37-51.

Scott, S. L., 2000. Bayesian methods for hidden Markov models: Recursive computing in the 21st century. Journal of the American Statistical Association 97, 337-351.

SIAM News. (2000). The Best of the 20th Century: Editors Name Top 10 Algorithms, Vol. 33, No. 2000. Download from http://www.siam.org/pdf/news/637.pdf.

Sorensen, D., and Gianola, D. (2002).Likelihood, Bayesian and MCMC Methods in Quantitative Genetics.New York: Springer, USA.

Tran, H. D. (2007). Investigation of deterioration models for stormwater pipe systems. School of Architectural, Civil and Mechanical Engineering, Victoria University, Australia.

Tran, H. D., Perera, B., and NG, A. (2009).“Comparison of Structural Deterioration Models for Stormwater Drainage Pipes” Computor-aided Civil and Infrastrucure Engineering, 24, 145-156.

Veshosky, D., and Beidleman, C. (1996).Closure to "Comparative Analysis of Bridge Superstructure Deterioration” by David Veshosky and Carl R. Beidleman. J. Struct. Eng. 122(6), 710-71.

Yin, F., Mou, J., Qiu, J. (2011). "A Bayesian MCMC Approach to Study the Safety of Vessel Traffic." ICTIS 2011, pp. 1838-1847.

Zhang, J., Wan, C., and Sato, T. (2013). "Advanced markov Chain Monte Carlo Approach for Finite Element Calibration under Uncertainty" Computor-aided Civil and Infrastrucure Engineering, 28, 522-530. 
Table 1 Chi-square values of three deterioration models

\begin{tabular}{|c|c|c|}
\hline \multirow{2}{*}{ Method } & \multicolumn{2}{|c|}{ Chi-square values with 4 Degree of freedoms $\left(\leq \chi_{0.05,4}^{2}=9.49\right)$} \\
\hline & Calibrated Dataset & Test dataset \\
\hline Regression Based optimization & 1.467 & 2.54 \\
\hline Bayesian maximum likelihood & 0.418 & 1.531 \\
\hline MCMC simulation with MHA & 0.268 & 1.508 \\
\hline
\end{tabular}

Table 2 Main Transition probability values with $95 \%$ confidence intervals

\begin{tabular}{ccccc}
\hline$p_{i i}$ & Mean & STD & Upper limit & Lower limit \\
\hline$p_{11}$ & 0.864 & 0.02564 & 0.867 & 0.860 \\
$p_{22}$ & 0.921 & 0.01651 & 0.923 & 0.918 \\
$p_{33}$ & 0.923 & 0.0178 & 0.925 & 0.920 \\
$p_{44}$ & 0.913 & 0.0195 & 0.915 & 0.910 \\
\hline
\end{tabular}


Figure 01

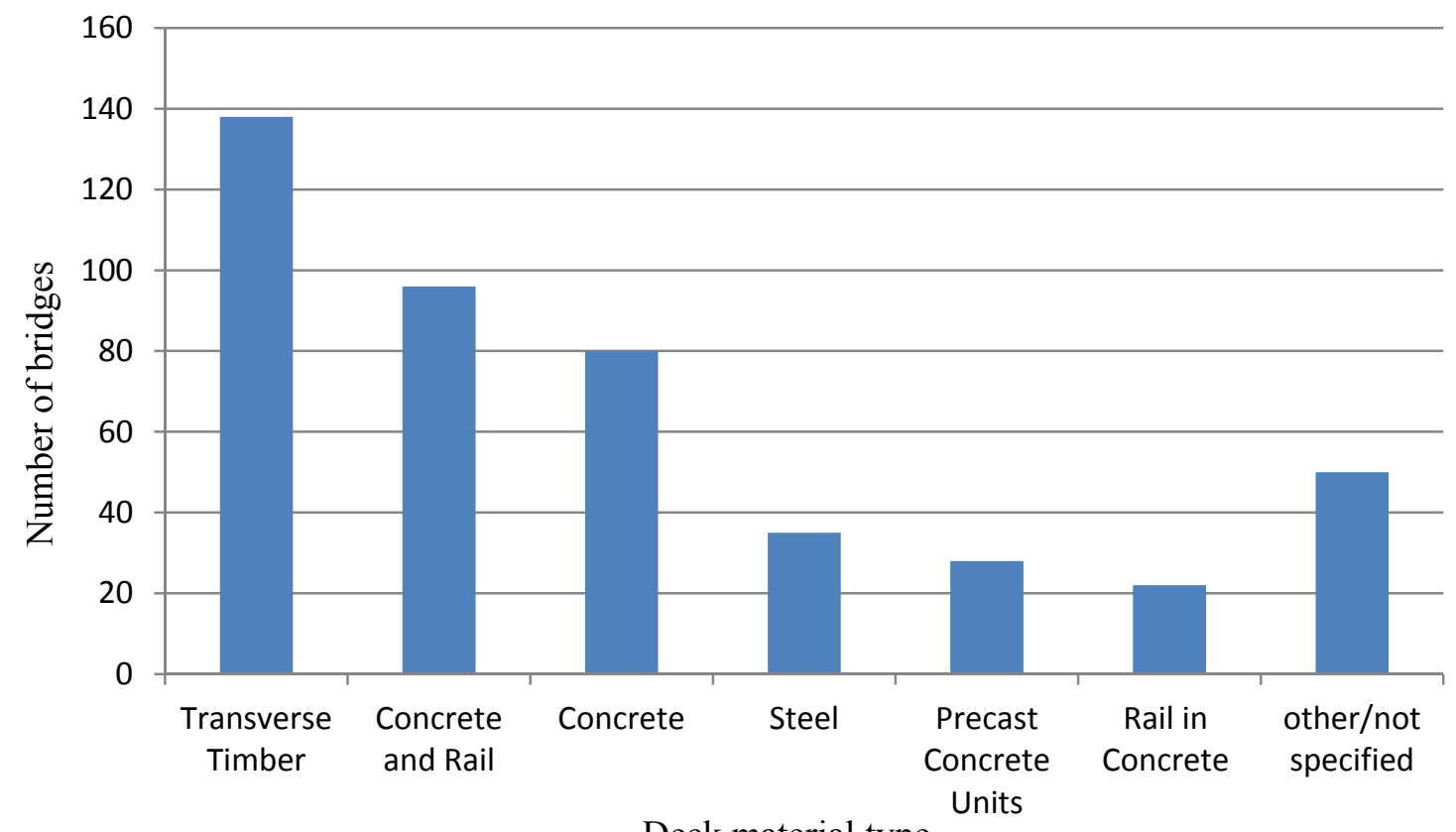

Deck material type 
Click here to download Figure: fig2.pdf

Figure 2

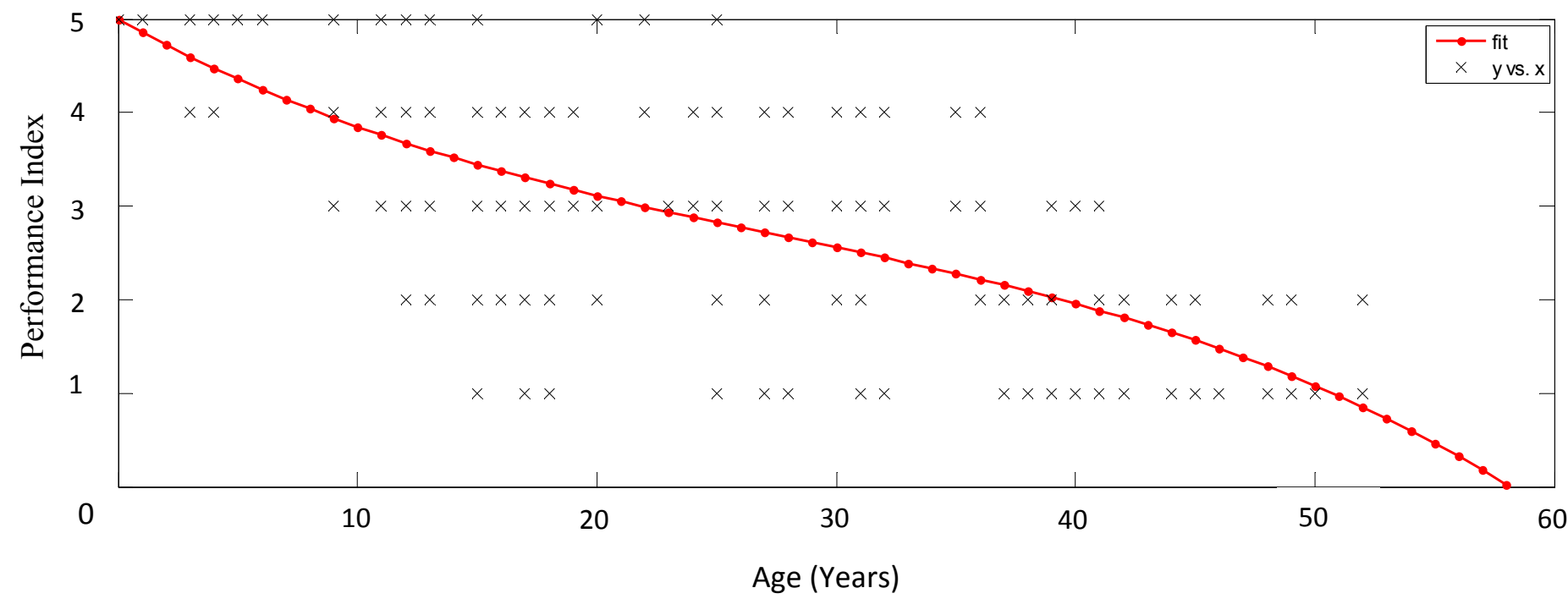



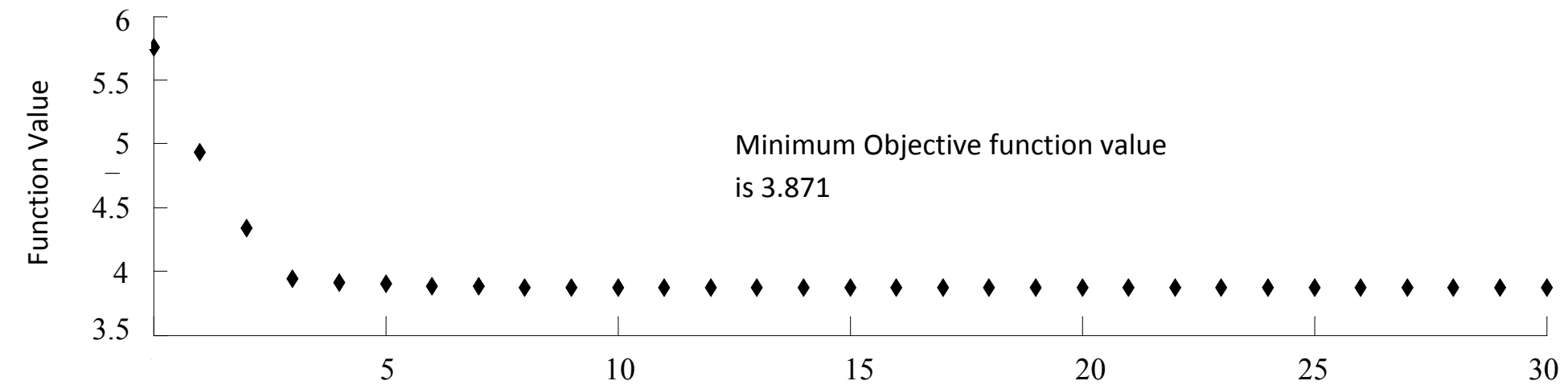

Number of Iterations 
Click here to download Figure: fig4.pdf

Figure 4

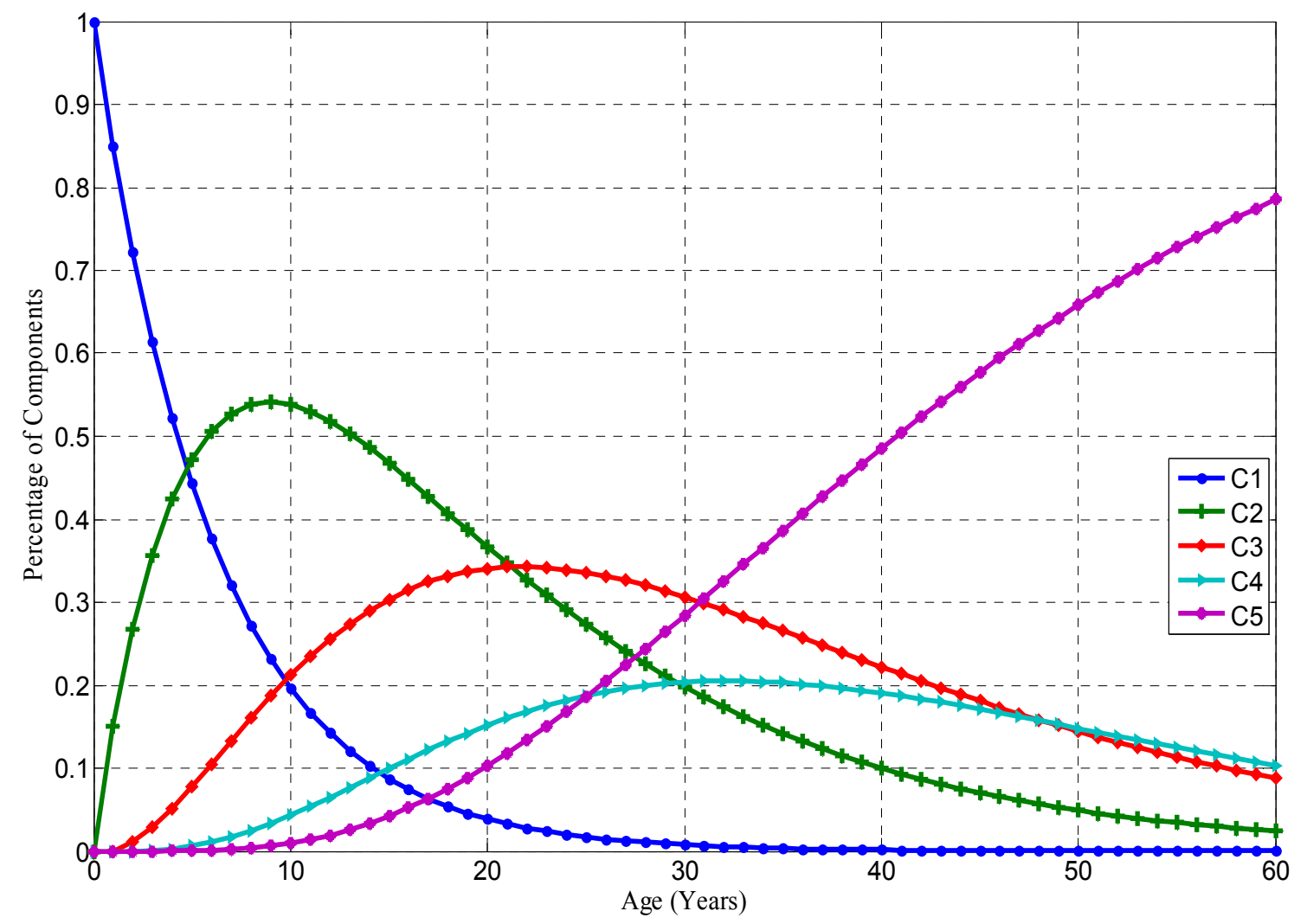


Figure 5
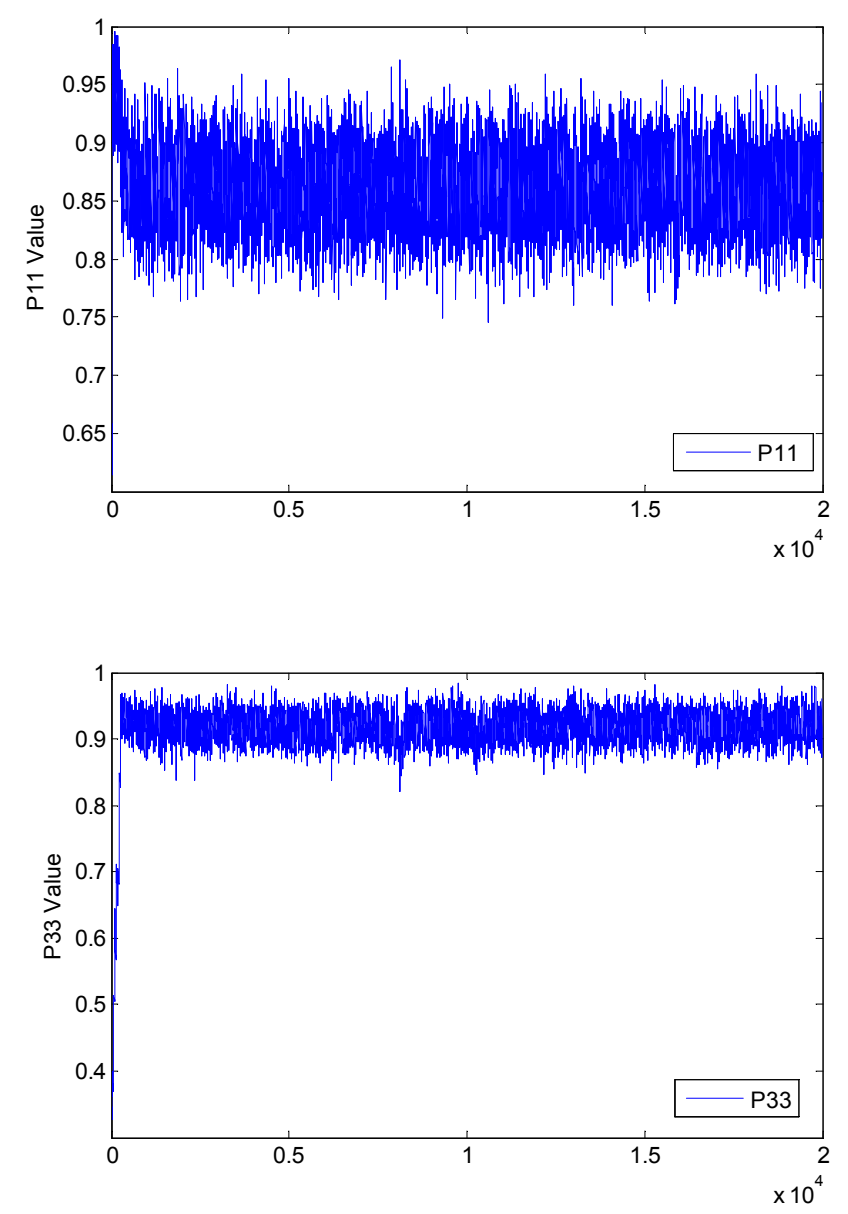
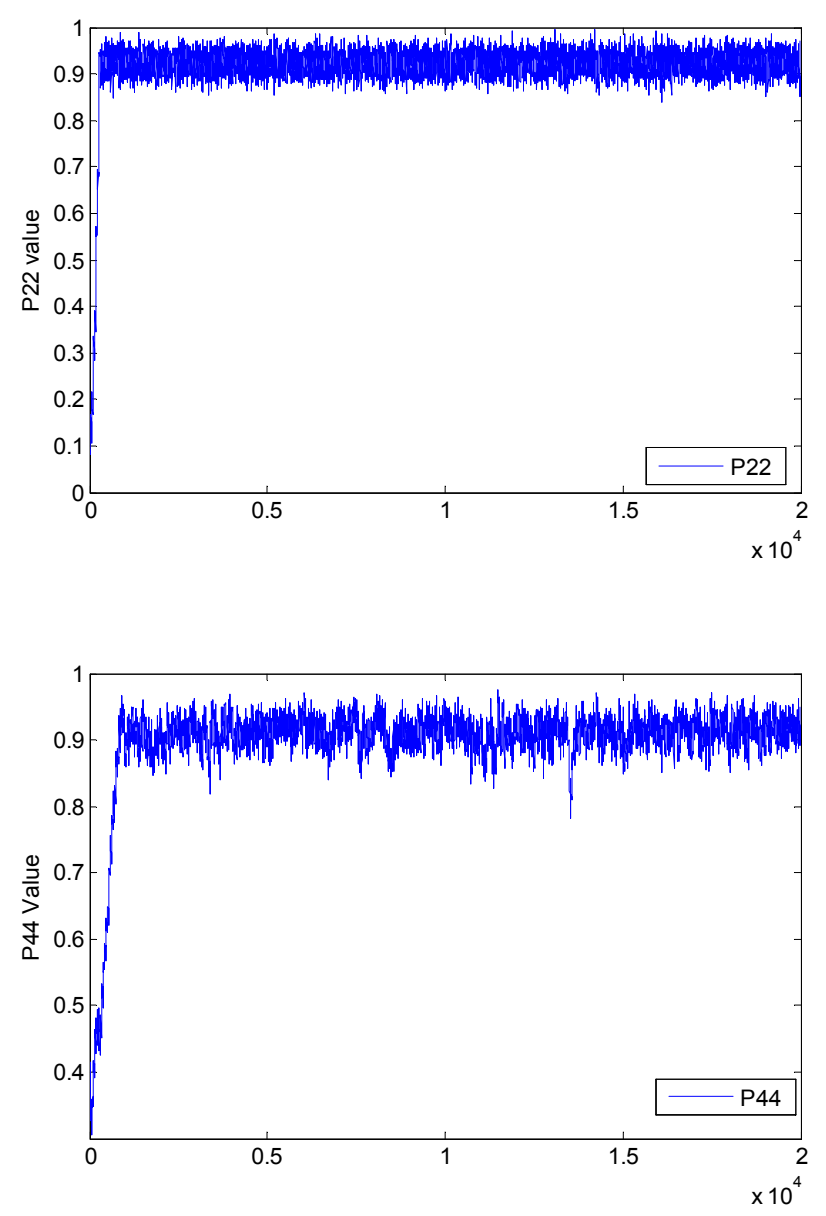
Figure 6
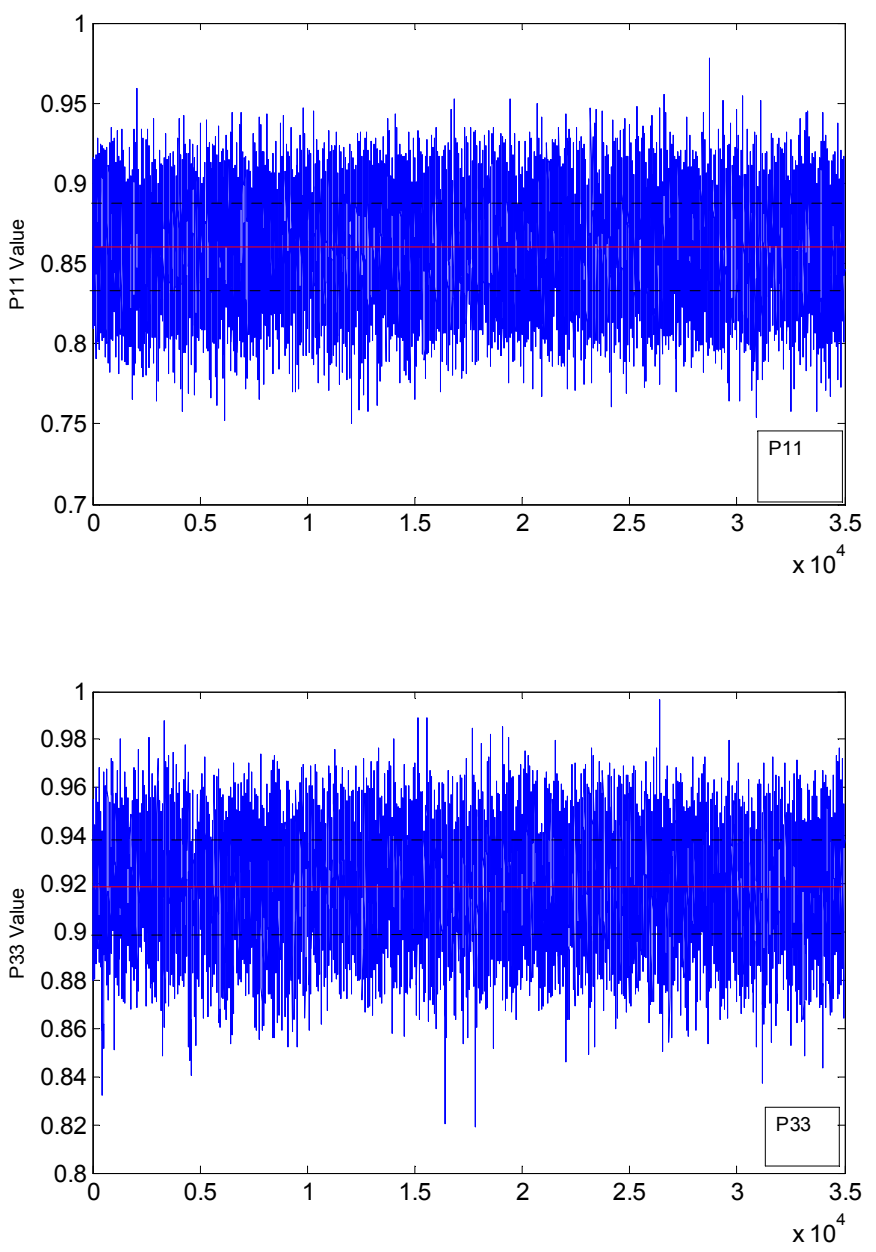
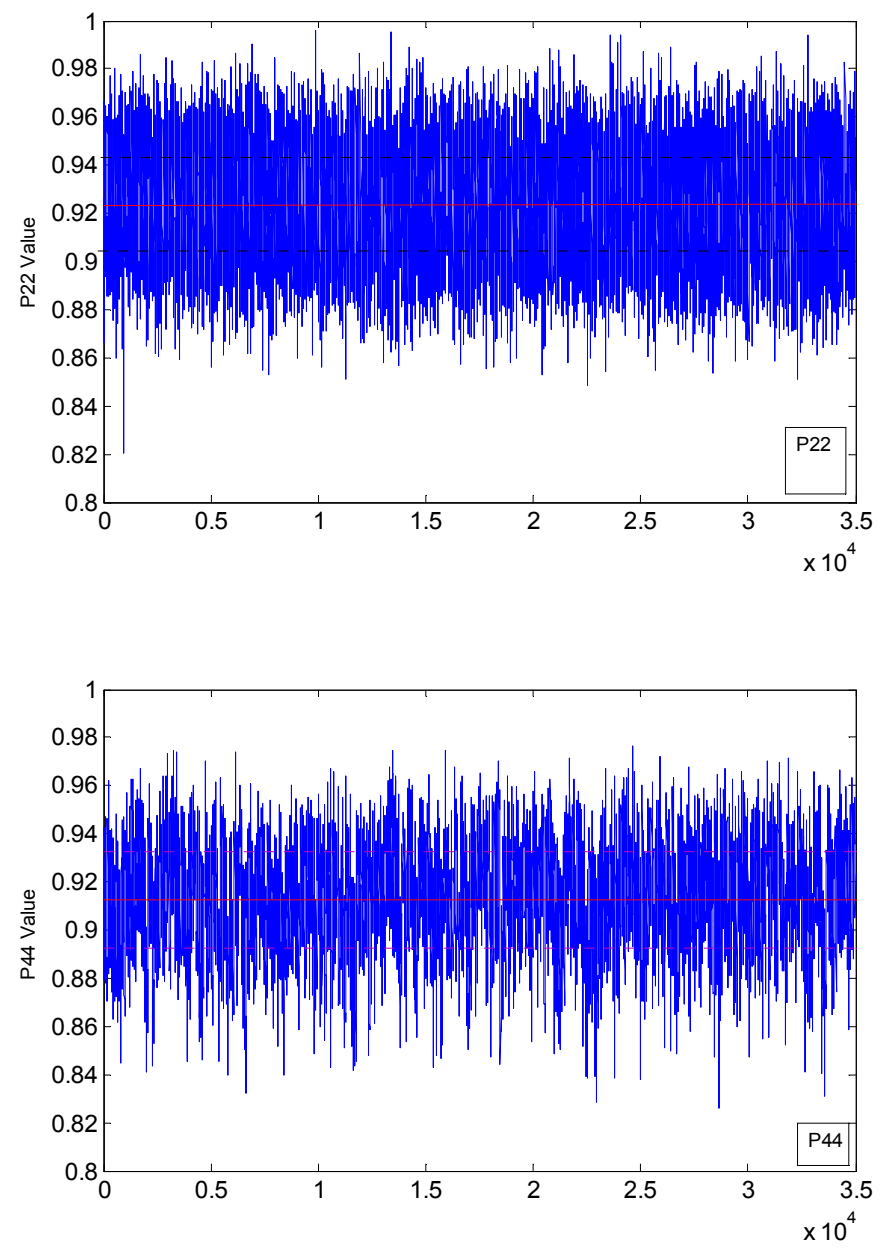
Figure 7
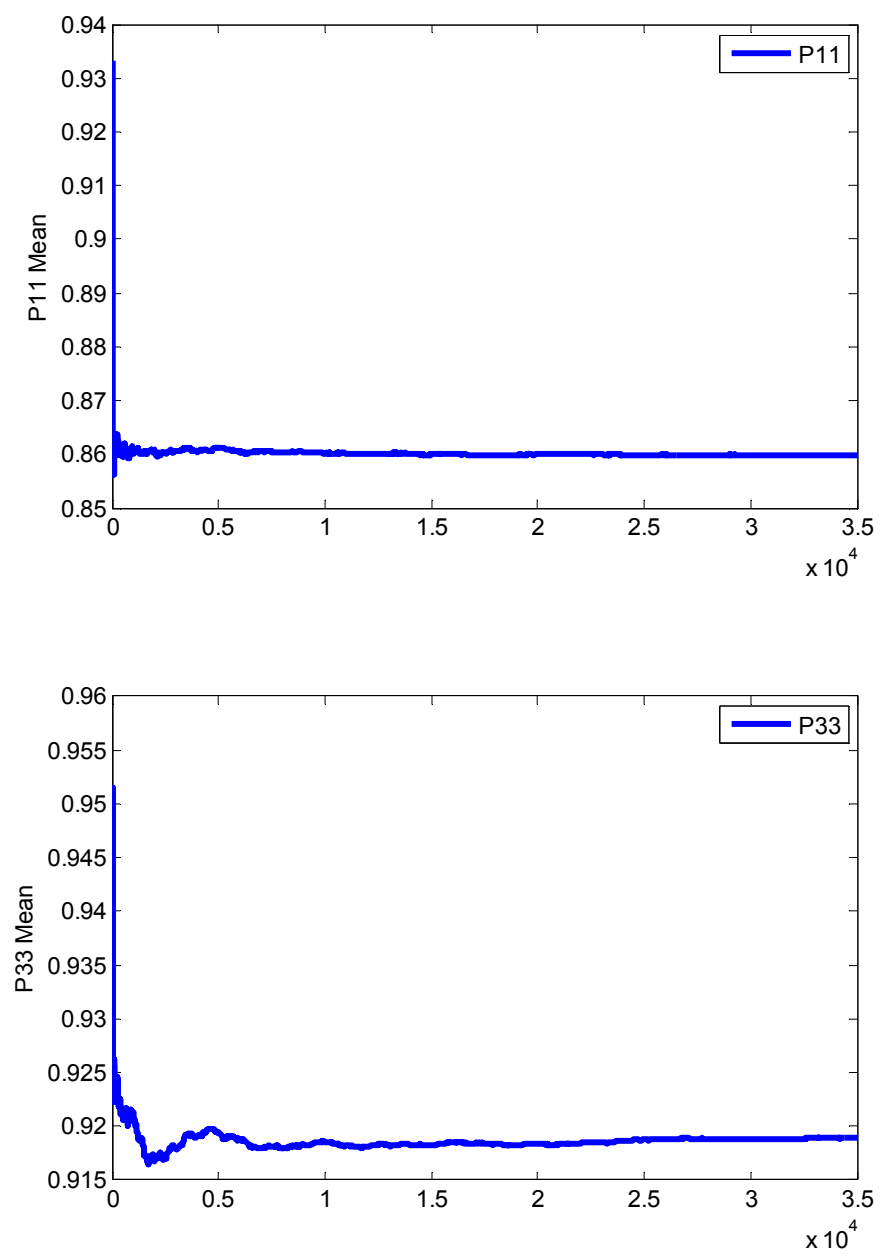
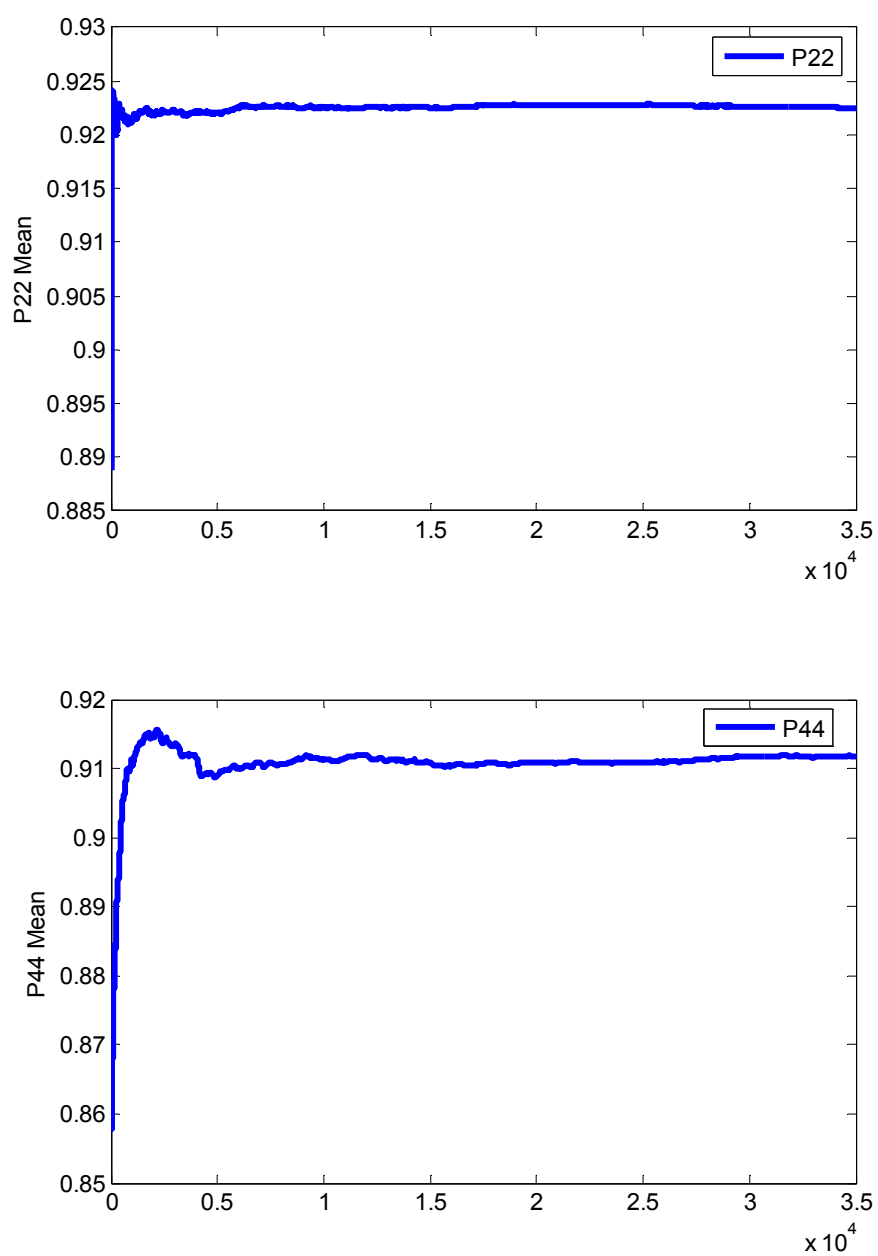
Figure 8
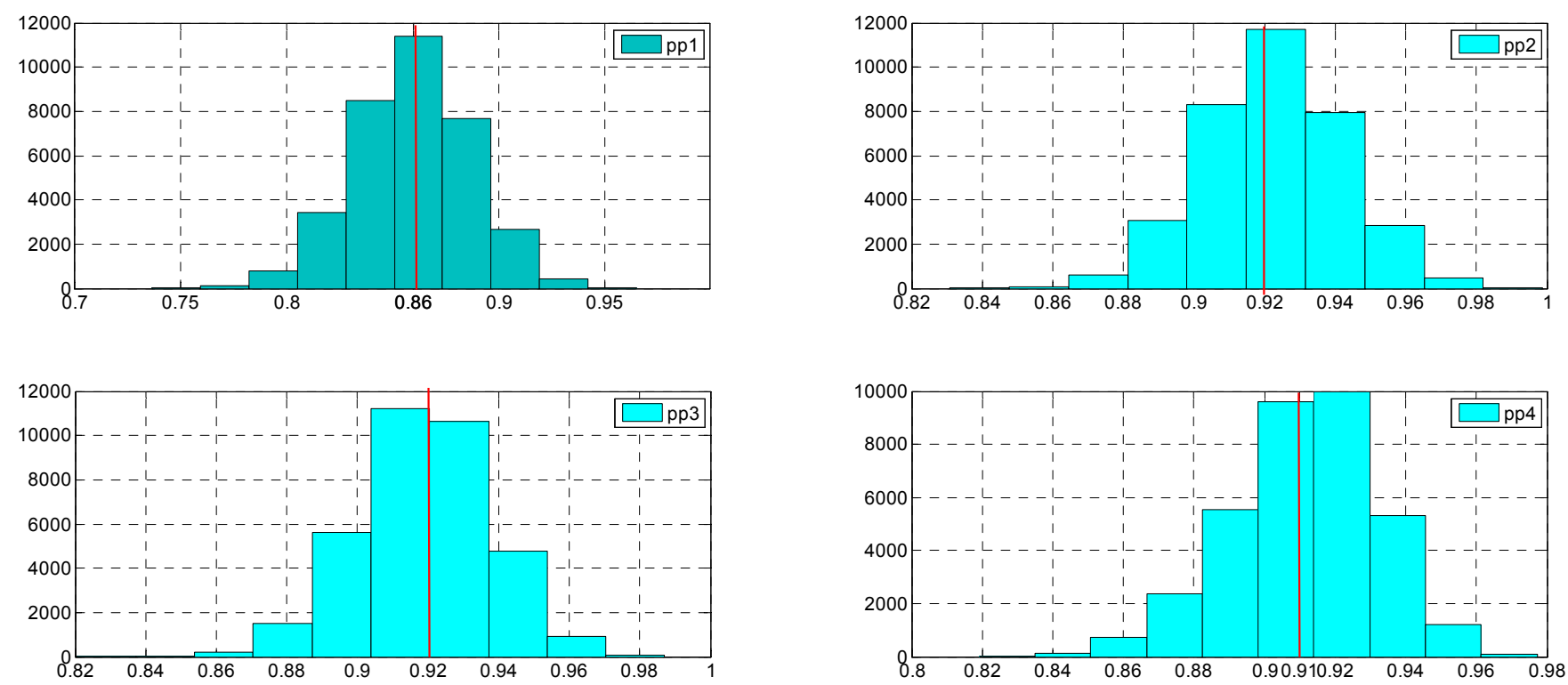
Figure 9
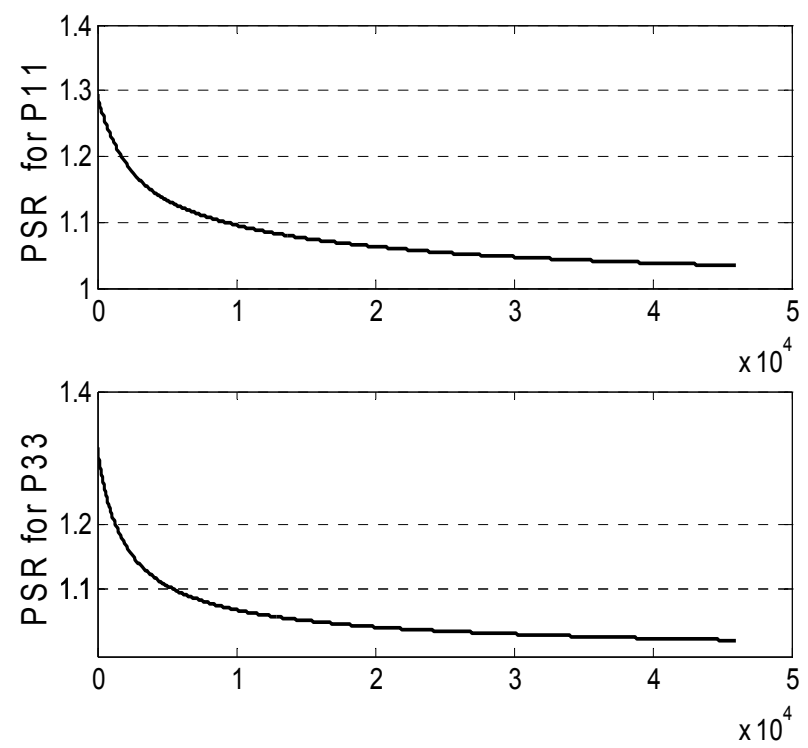
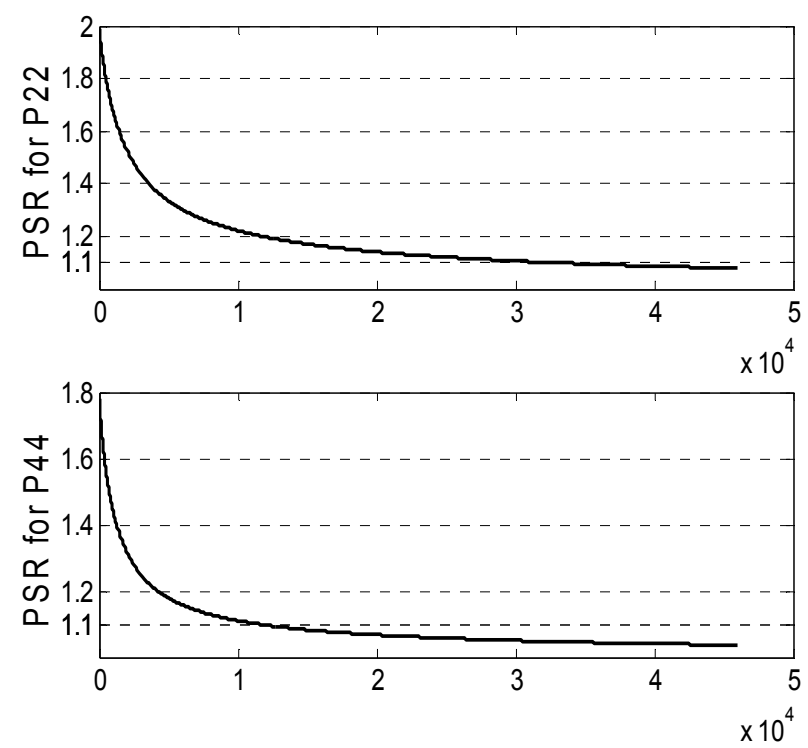
Click here to download Figure: fig10.pdf

Figure 10

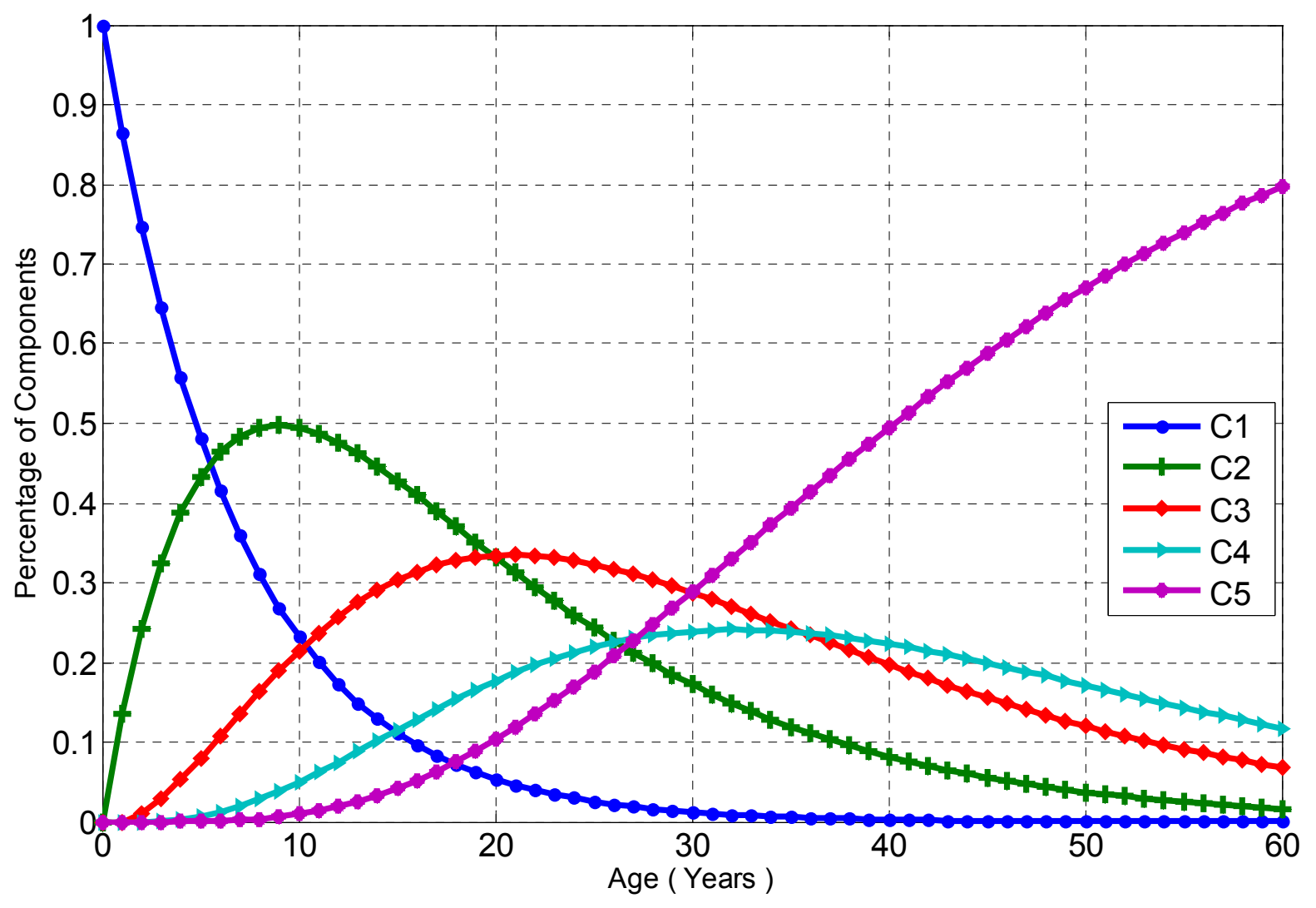


Figure captions

Figure 1 Bridge classification according deck material type

Figure 2 Regression $3^{\text {rd }}$ polynomial curve fitting analysis result for given data set

Figure 3 Nonlinear optimization function values for transition probabilities in RNO

Figure 4 Condition Percentage distribution of each condition state vs. age in RNO

Figure 5 Trace plots after 20000 iterations with no warm-up runs for $p_{11}, p_{22}, p_{33}$ and $p_{44}$

Figure 6 Trace plots for 50000 iterations with 15000 warm-up runs for $p_{11}, p_{22}, p_{33}$ and $p_{44}$

Figure 7 Mean value trace plots for 50000 iterations with 15000 warm-up runs for $p_{11}, p_{22}, p_{33}$ and $p_{44}$

Figure 8 Generated sample posterior distributions of $p_{11}, p_{22}, p_{33}$ and $p_{44}$

Figure 9 Convergence Diagnostic characteristics of Potential Scale Reduction factors for for $p_{11}, p_{22}$, $p_{33}$ and $p_{44}$ from 2000 to 50000 iterations

Figure 10 Condition Percentage distribution of each condition state $v s$. age in MCMC with MHA for entire data set

\section{$\underline{\text { Table captions }}$}

Table 1 Chi-square values of three deterioration models

Table 2 Main Transition probability values with 95\% confidence intervals 